\title{
Heisenberg Algebra in the Bargmann-Fock Space with Natural Cutoffs
}

\author{
Maryam Roushan ${ }^{1}$ and Kourosh Nozari \\ ${ }^{1}$ Department of Physics, Faculty of Basic Sciences, Islamic Azad University, Shahreza Branch, Shahreza, Iran \\ ${ }^{2}$ Department of Physics, Faculty of Basic Sciences, University of Mazandaran, P.O. Box 47416-95447, Babolsar, Iran
}

Correspondence should be addressed to Maryam Roushan; m57.roshan@gmail.com

Received 4 September 2013; Revised 9 November 2013; Accepted 9 November 2013; Published 2 February 2014

Academic Editor: Elias C. Vagenas

Copyright ( 2014 M. Roushan and K. Nozari. This is an open access article distributed under the Creative Commons Attribution License, which permits unrestricted use, distribution, and reproduction in any medium, provided the original work is properly cited. The publication of this article was funded by SCOAP ${ }^{3}$.

We construct a Heisenberg algebra in Bargmann-Fock space in the presence of natural cutoffs encoded as minimal length, minimal momentum, and maximal momentum through a generalized uncertainty principle.

\section{Introduction: The Generalized Uncertainty Principle and Fuzzy Spacetime}

According to the equivalence principal in general relativity, gravitational field is coupled to everything. This means that photons in Heisenberg gedankenexperiment are actually coupled with electrons gravitationally and this leads to modification of the standard uncertainty principle. It has been characterized that gravity in very small length scales causes serious change in the structure of spacetime. It causes minimal uncertainty in positions of atomic and subatomic particles [1-15]. In fact, there is absolutely smallest uncertainty in position measurement of any quantum mechanical system and this feature leads nontrivially to the existence of a minimal measurable length in the order of Planck length. Existence of this natural cutoff requires deformation of the standard Heisenberg uncertainty principle to the so-called generalized uncertainty principle (GUP) (see, for instance, $[13,14,16-20])$. In one dimension of position and momentum operators, the deformed Heisenberg algebra can be represented as

$$
[X, P]=i \hbar\left(1+\beta P^{2}\right)
$$

In general, for two symmetric operators $A$ and $B$, we have

$$
\Delta A \Delta B \geq|\langle[A, B]\rangle| .
$$

So the generalized uncertainty principle can be deduced as

$$
\Delta X \Delta P \geq \frac{\hbar}{2}\left[1+\beta(\Delta P)^{2}\right]
$$

While in ordinary quantum mechanics $\Delta X$ can be made arbitrarily small by letting $\Delta P$ grows correspondingly, this is no longer the case if (3) holds. If for decreasing $\Delta X, \Delta P$ increases, the new term $\beta(\Delta P)^{2}$ on the right hand side of (3) will eventually grow faster than the left hand side. Hence $\Delta X$ can no longer be made arbitrarily small $[16,18]$. To obtain this minimal uncertainty, we saturate inequality in (3) and solve the resulting equation for $\Delta P$,

$$
\Delta P=\frac{\Delta X \pm \sqrt{(\Delta X)^{2}-\hbar^{2} \beta}}{\hbar \beta} .
$$

The reality of solutions requires positivity of the term in square root, leading to

$$
(\Delta X)_{0}=\hbar \sqrt{\beta} .
$$

This being the smallest uncertainty in position measurement leads nontrivially to the existence of a minimal measurable length. In fact, a key characteristic of quantum theory is the emergence of uncertainties, and one might expect that the distance observable would also be affected by 
uncertainties. Actually, various heuristic arguments suggest that for such a distance observable the uncertainties might be more pervasive; in ordinary quantum theory one is still able to measure sharply any given observable, though at the cost of renouncing all information on a conjugate observable, but it appears plausible that a quantum-gravity distance observable would be affected by irreducible uncertainties. Quantum gravity suggests that in the Planck-scale regime there should be some absolute limitations on the measurability of distances. This restricted resolution of spacetime structure is referred to as spacetime fuzziness "foamy or fractal spacetime" [21]. This picture replaces point-like structures with a smeared, distributional structure. The effect of smearing could be mathematically implemented as a substitution rule; the Dirac-delta function representing position of point-like particles is replaced everywhere with a Gaussian distribution with minimal width of the order of the Planck length.

On the other hand, in the context of the Doubly special relativity (DSR) theories (for review see [22-27]), one can show that a test particle's momentum cannot be arbitrarily imprecise. In fact, there is an upper bound for momentum fluctuations [28-31]. As a nontrivial assumption, this may lead to a maximal measurable momentum for a test particle (see $[20,32-34])$. In this framework, the GUP that predicts both minimal observable length and maximal momentum can be written (with $\hbar=1$ ) as follows [32, 33]:

$$
\begin{gathered}
\Delta X \Delta P \geq \frac{1}{2}\left[1+\left(\frac{\alpha}{\sqrt{\left\langle P^{2}\right\rangle}}+4 \alpha^{2}\right)(\Delta P)^{2}\right. \\
\left.+4 \alpha^{2}\langle P\rangle^{2}-2 \alpha \sqrt{\left\langle P^{2}\right\rangle}\right] .
\end{gathered}
$$

Since $(\Delta P)^{2}=\left\langle P^{2}\right\rangle-\langle P\rangle^{2}$, by setting $\langle P\rangle=0$ to obtain absolute minimal length, we find

$$
\Delta X \Delta P \geq \frac{1}{2}\left[1-\alpha(\Delta P)+4 \alpha^{2}(\Delta P)^{2}\right] .
$$

This GUP contains both a minimal length and a maximal momentum. To see how a maximal momentum arises in this setup (see [20] for details), we note that with GUP (7) the absolute minimal measurable length is given by $\Delta X_{\min }(\langle P\rangle=$ $0) \equiv \Delta X_{0}=3 \alpha / 2$. Due to duality of position and momentum operators, it is reasonable to assume $\Delta X_{\min } \propto \Delta P_{\max }$. By saturating the inequality in relation (7), we find

$$
2(\Delta X \Delta P)=\left(1-\alpha(\Delta P)+4 \alpha^{2}(\Delta P)^{2}\right) .
$$

This results in

$$
(\Delta P)^{2}-\frac{(2 \Delta X+\alpha)}{4 \alpha^{2}} \Delta P+\frac{1}{4 \alpha^{2}}=0 .
$$

So we obtain

$$
\left(\Delta P_{\max }\right)^{2}-\frac{\left(2 \Delta X_{\min }+\alpha\right)}{4 \alpha^{2}} \Delta P_{\max }+\frac{1}{4 \alpha^{2}}=0 .
$$

Now by using the value of $\Delta X_{\min }$, we find

$$
\left(\Delta P_{\max }\right)^{2}-\frac{1}{\alpha} \Delta P_{\max }+\frac{1}{4 \alpha^{2}}=0 .
$$

The solution of this equation is

$$
\Delta P_{\max }=\frac{1}{2 \alpha} .
$$

So, there is an upper bound on particle's momentum uncertainty. As a nontrivial assumption, we assume that this maximal uncertainty in particle's momentum is indeed the maximal measurable momentum. This is of the order of Planck momentum.

After introducing minimal length and maximal momentum as natural cutoffs and also introduction of the notion of spacetime fuzziness, we introduce another cutoff, the minimal momentum. It is known that for large distances, where the curvature of space-time becomes important, there is no notion of a plane wave on a general curved spacetime [17] (see also [35]). This means that there appears a limit to the precision with which the corresponding momentum can be described. One can express this as a nonzero minimal uncertainty in momentum measurement. In this framework, we define new GUP with minimal length, minimal momentum, and maximal momentum as follows:

$$
\Delta X \Delta P \geq \frac{\hbar}{2}\left(1+\alpha(\Delta X)^{2}-\sqrt{\beta}(\Delta P)+\beta(\Delta P)^{2}\right),
$$

where $\beta$ is a positive constant. By saturating this inequality and solving the resulting equation, we obtain $\Delta P$ as

$$
\begin{aligned}
\Delta P= & \frac{(2 \Delta X+\hbar \sqrt{\beta})}{2 \hbar \beta} \\
& \pm \frac{\sqrt{(2 \Delta X+\hbar \sqrt{\beta})^{2}-4 \hbar \beta\left[\hbar+\hbar \alpha(\Delta X)^{2}\right]}}{2 \hbar \beta} .
\end{aligned}
$$

So, the minimum uncertainty for position measurement is given by

$$
\Delta X_{\min }=\frac{\sqrt{\beta}}{2} \hbar,
$$

and minimum uncertainty for momentum measurement is

$$
\Delta P_{\text {min }}=\sqrt{\alpha} \hbar .
$$

Now by setting the value of $\Delta X_{\min }$ in (14), we attain the maximum uncertainty for momentum measurement as follows:

$$
\Delta P_{\max }=\frac{1}{\sqrt{\beta}}\left(1+\frac{\hbar^{2} \alpha \beta}{4}\right)^{1 / 2} .
$$

Thus we have shown that the uncertainty relation (13) encodes properly the existence of natural cutoffs. 


\section{Hilbert Space Representation with Natural Cutoffs}

There are distinct approaches toward quantum gravity that all imply the presence of an observable minimal length belonging to the Planck length category. The minimal length makes serious problems in representation in the coordinate space of quantum mechanics. In case the minimal momentum is not taken into consideration, the representation of the momentum space would be sufficient to formulate the Hilbert space. But, whenever the minimal momentum is accounted for, the representation of the momentum space would lose the credibility it has in the standard quantum mechanics. Hence, modifications in Hilbert space representation with the help of natural cutoffs seem to be necessary. So far, the formulation of the Hilbert space has been done separately based on the minimal length [16], minimal length and minimal momentum [17], and minimal length and maximal momentum [20]. The present paper aims to simultaneously treat the Hilbert space in the presence of all natural cutoffs, that is, the minimal length, the minimal momentum, and the maximal momentum, and the consequences are to be reviewed as well. This is going to be done through a new, generalized Hilbert space called the Bargmann-Fock space that includes $q$-algebraic variables.

2.1. Heisenberg Algebra with Natural Cutoffs. Hinrichsen and Kempf in [17] defined the associative Heisenberg algebra with minimal length and minimal momentum addressed by the following commutation relation with $\alpha, \beta \geq 0$ :

$$
[X, P]=i \hbar\left(1+\alpha X^{2}+\beta P^{2}\right) \text {. }
$$

Here we add a new ingredient: the existence of a maximal measurable momentum. With this extra ingredient, the associative Heisenberg algebra in the presence of all natural cutoffs contains the following commutation relation:

$$
[X, P]=i \hbar\left(1+\alpha X^{2}-\sqrt{\beta} P+\beta P^{2}\right) .
$$

We are going to use the platform of [17] in our setup. For this purpose, we transform (19) in a manner that is comparable with (18) (or equation (2) of [17]). In this viewpoint, (19) can be rewritten as follows:

$$
[X, P]=i \hbar\left[\frac{3}{4}+\alpha X^{2}+\beta\left(P-\frac{1}{2 \sqrt{\beta}}\right)^{2}\right] .
$$

The importance of this commutation relation lies in the fact that it contains all natural cutoffs. In fact, both UV and IR sectors of the underlying quantum theory are addressed properly in this commutation relation. By comparing (18) and (20), we see that these two relations are related through the transformations

$$
\begin{gathered}
P \longrightarrow P-\frac{1}{2 \sqrt{\beta}}, \\
1 \longrightarrow \frac{3}{4} .
\end{gathered}
$$

So, the mathematical framework of Hinrichsen-Kempf pioneer work [17] can be applied to the present problem. We note that when one considers both minimal length and minimal momentum hypothesis, representation of position and momentum spaces breaks down. In this situation, there is no continuous Hilbert space representation and we have to build a generalized Hilbert space representation as follows.

2.2. Heisenberg Algebra in Bargmann-Fock Space. Existence of natural cutoffs requires a generalized Heisenberg algebra in Fock space developed in the context of quantum groups. In this framework, due to the fundamental structure of spacetime, all operators are anticommutative. In BargmannFock space the following relations for $\alpha$ and $\beta$ hold:

$$
\begin{aligned}
& \beta=\frac{L\left(q^{2}-1\right)}{K\left(q^{2}+1\right)}, \\
& \alpha=\frac{K\left(q^{2}-1\right)}{L\left(q^{2}+1\right)},
\end{aligned}
$$

where the constants $L, K$ carry units of length and momentum and are related by

$$
4 K L=\hbar\left(1+q^{2}\right)
$$

and $q$ is the deformation parameter. Based on the deformed algebra in Fock space, we obtain the commutation relation with minimal length, minimal momentum, and maximal momentum, as follows:

$$
[X, P]=i \hbar\left[1+\frac{\left(q^{2}-1\right)}{4 L^{2}} X^{2}-\sqrt{\frac{q^{2}-1}{4 K^{2}}} P+\frac{\left(q^{2}-1\right)}{4 K^{2}} P^{2}\right],
$$

or through (21) and (22),

$$
[X, P]=i \hbar\left[\frac{3}{4}+\left(q^{2}-1\right)\left(\frac{X^{2}}{4 L^{2}}+\frac{\left(P-K / \sqrt{q^{2}-1}\right)^{2}}{4 K^{2}}\right)\right] .
$$

Note that these transform to ordinary quantum mechanics results where we set $q=1$. The corresponding uncertainty relation is as follows:

$$
\begin{aligned}
& \Delta X \Delta\left(p-\frac{1}{2 \sqrt{\beta}}\right) \\
& \geq \frac{\hbar}{2}\left[\frac{3}{4}+\alpha\left((\Delta X)^{2}+\langle X\rangle^{2}\right)\right. \\
&\left.\quad+\beta\left(\left(\Delta\left(P-\frac{1}{2 \sqrt{\beta}}\right)\right)^{2}+\left\langle P-\frac{1}{2 \sqrt{\beta}}\right\rangle^{2}\right)\right],
\end{aligned}
$$


or simply as

$$
\begin{aligned}
\Delta X \Delta P \geq \frac{\hbar}{2}[ & \frac{3}{4}+\alpha\left((\Delta X)^{2}+\langle X\rangle^{2}\right) \\
& \left.+\beta\left((\Delta P)^{2}+\left\langle P-\frac{1}{2 \sqrt{\beta}}\right\rangle^{2}\right)\right] .
\end{aligned}
$$

Based on this uncertainty relation, there are uncertainties in position and momentum as follows:

$$
\begin{aligned}
&\left(\Delta X_{\min }\right)^{2}= \frac{L^{2}\left(q^{2}-1\right)}{q^{2}} \\
& \times {\left[\frac{3}{4}+\left(q^{2}-1\right)\right.} \\
&\left.\times\left(\frac{\langle X\rangle^{2}}{4 L^{2}}+\frac{\left\langle P-K / \sqrt{q^{2}-1}\right\rangle^{2}}{4 K^{2}}\right)\right] \\
&\left.\left(\Delta P_{\min }\right)^{2}=\frac{K^{2}}{\left.q^{2}-1\right)}\right] {\left[\frac{3}{4}+\left(q^{2}-1\right)\right.} \\
&\left.\times\left(\frac{\langle X\rangle^{2}}{4 L^{2}}+\frac{\left\langle P-K / \sqrt{q^{2}-1}\right\rangle^{2}}{4 K^{2}}\right)\right]
\end{aligned}
$$

Note that $\Delta P_{\max }$ can be obtained through the procedure adopted in Section 1 .

\section{Some Analysis on Maximal \\ Localization States}

Now we consider the states $\left|\varphi_{x}^{\mathrm{ml}}\right\rangle$ of maximal localization around a position $x$ and we set the expectation value of the momentum to be zero

$$
\begin{aligned}
& \left\langle\psi_{x}^{\mathrm{ml}}|X| \psi_{x}^{\mathrm{ml}}\right\rangle=x, \\
& \left\langle\psi_{x}^{\mathrm{ml}}|P| \psi_{x}^{\mathrm{ml}}\right\rangle=0 .
\end{aligned}
$$

For maximal localization states in the presence of both minimal length and minimal momentum, we use the following equation $[16,17]$ :

$$
((X-\langle X\rangle)+i \beta(P-\langle P\rangle))\left|\psi_{x}^{\mathrm{ml}}\right\rangle=0
$$

We note that we used (21) to arrive at this relation, but the terms $1 / 2 \sqrt{\beta}$ have canceled each other in $P$ and $\langle P\rangle$. Now by setting $\beta$ in the above equation, we have

$$
\left(\frac{q^{2}+1}{L}(X-\langle X\rangle)+i \frac{q^{2}-1}{K} P\right)\left|\psi_{x}^{\mathrm{ml}}\right\rangle=0 .
$$

Adding the existence of maximal momentum as a new ingredient through transformation of (21) and (22), we find

$$
\begin{aligned}
& \left(\frac{q^{2}+1}{L}(X-\langle X\rangle)+i \frac{q^{2}-1}{K}\left(P-\frac{K}{\sqrt{q^{2}-1}}\right)\right)\left|\psi_{x}^{\mathrm{ml}}\right\rangle \\
& \quad=0 .
\end{aligned}
$$

3.1. Maximal Localization States in Bargmann-Fock Space. We consider the states $\left|\varphi_{x}^{\mathrm{ml}}\right\rangle$ to be maximally localized around a position $x$. Following [17], to calculate these states, we expand the $\left|\varphi_{x}^{\mathrm{ml}}\right\rangle$ based on $q$-Hermite polynomials in Fock basis

$$
\left|\varphi_{x}^{\mathrm{ml}}\right\rangle=\frac{1}{N(x)} \sum_{n=0}^{\infty} q^{-3 n / 2} c_{n}(x)|n\rangle,
$$

where $N(x)$ is the normalization factor defined as follows:

$$
N(x)=\sum_{n=0}^{\infty} q^{-3 n} c_{n}^{2}(x) .
$$

Now by setting momentum and position operators in (32), we obtain a new relation in the Fock representation as

$$
\begin{aligned}
& {\left[\left(q^{2}+1\right)\left(a^{\dagger}+a-\frac{x}{L}\right)-\left(q^{2}-1\right)\left(a^{\dagger}-a\right)\right.} \\
& \left.-i\left(\sqrt{q^{2}-1}\right)\right]\left|\psi_{x}^{\mathrm{ml}}\right\rangle=0,
\end{aligned}
$$

where we have used the following relations:

$$
\begin{aligned}
& X=L\left(a^{\dagger}+a\right), \\
& P=i K\left(a^{\dagger}-a\right),
\end{aligned}
$$

to define raising and lowering operators in Fock space. Such that $a$ and $a^{\dagger}$ obey generalized commutation relations [17]

$$
a a^{\dagger}-q^{2} a^{\dagger} a=1
$$

Now by using (33) to solve (35), we obtain the following recursion relation

$$
\begin{aligned}
& {\left[\frac{q+q^{-1}}{2 L} x+\frac{\sqrt{q^{-2}-1}}{2}\right] c_{n}(x)} \\
& \quad=\sqrt{q^{-1}[n+1]} c_{n+1}+\sqrt{q[n]} c_{n-1}(x),
\end{aligned}
$$


subject to the following boundary conditions:

$$
c_{0}(x)=1, \quad c_{-1}(x)=0,
$$

where

$$
[n] \equiv \frac{q^{2 n}-1}{q^{2}-1} .
$$

Comparing this result with the corresponding relation obtained by Hinrichsen and Kempf in [17] in the presence of minimal length and minimal momentum, we see that incorporation of the maximal momentum results in the extra term were given as $\left(\sqrt{q^{-2}-1} / 2\right) c_{n}(x)$ in the recursion relation. This is the main deference of our setup with HinrichsenKempf framework. The coefficients $c_{n}(x)$ are given by $q-$ Hermit polynomials [36] as follows:

$$
\begin{aligned}
c_{n}(x)= & \left(1+\frac{\left(q^{2}-1\right)\left(q^{-2}-1\right)}{4 \omega x}\right)^{n} \\
& \times \sqrt{\frac{q^{n}}{\left(q^{2}-1\right)^{n}[n] !}} i^{-n} H_{n}\left(i \omega x \mid q^{2}\right) .
\end{aligned}
$$

Having $c_{n}(x)$, the maximally localized states are given by (33). This gives the complete structure of generalized Hilbert space in the presence of all natural cutoffs.

\section{Summary}

Representation of states in quantum mechanics, in the presence of quantum gravity induced natural cutoffs, is an important issue. So far this issue has been studied separately in the presence of minimal length [16], minimal length and minimal momentum [17], and minimal length and maximal momentum [20]. In [37], the authors have considered the Hilbert space representation in the presence of all natural cutoffs, simultaneously. Here we complete this study by further investigation of the scenario and its consequences with more details. This has been done through introduction of a generalized Hilbert space and the Bargmann-Fock space that includes $q$-deformed algebraic variables.

\section{Conflict of Interests}

The authors declare that there is no conflict of interests regarding the publication of this paper.

\section{References}

[1] G. Veneziano, "A stringy nature needs just two constants," Europhysics Letters, vol. 2, no. 3, article 199, 1986.

[2] D. Amati, M. Cialfaloni, and G. Veneziano, "Superstring collisions at planckian energies," Physics Letters B, vol. 197, no. 1-2, pp. 81-88, 1987.

[3] D. Amati, M. Cialfaloni, and G. Veneziano, "Can spacetime be probed below the string size?” Physics Letters B, vol. 216, no. 1-2, pp. 41-47, 1989.
[4] D. J. Gross and P. F. Mende, “The high-energy behavior of string scattering amplitudes," Physics Letters B, vol. 197, no. 1-2, pp. 129134, 1987.

[5] K. Konishi, G. Paffuti, and P. Provero, "Minimum physical length and the generalized uncertainty principle in string theory," Physics Letters B, vol. 234, no. 3, pp. 276-284, 1990.

[6] R. Guida, K. Konishi, and P. Provero, "On the short distance behavior of string theories," Modern Physics Letters A, vol. 6, no. 16, pp. 1487-1504, 1991.

[7] M. Kato, "Particle theories with minimum observable length and open string theory," Physics Letters B, vol. 245, no. 1, pp. 4347, 1990.

[8] L. J. Garay, "Quantum gravity and minimal length," International Journal of Modern Physics A, vol. 10, no. 2, pp. 145-166, 1995.

[9] S. Capozziello, G. Lambiase, and G. Scarpetta, "Generalized uncertainty principle from quantum geometry," International Journal of Theoretical Physics, vol. 39, no. 1, pp. 15-22, 2000.

[10] M. Maggiore, "A generalized uncertainty principle in quantum gravity," Physics Letters B, vol. 304, no. 1-2, pp. 65-69, 1993.

[11] M. Maggiore, "The algebraic structure of the generalized uncertainty principle," Physics Letters B, vol. 319, no. 1-3, pp. 83-86, 1993.

[12] M. Maggiore, "Quantum groups, gravity, and the generalized uncertainty principle," Physical Review D, vol. 49, no. 10, pp. 5182-5187, 1994.

[13] S. Hossenfelder, "Minimal length scale scenarios for quantum gravity," Living Reviews in Relativity, vol. 16, 2013.

[14] S. Hossenfelder, "Can we measure structures to a precision better than the Planck length?" Classical and Quantum Gravity, vol. 29, no. 11, Article ID 115011, 2012.

[15] F. Scardigli, "Generalized uncertainty principle in quantum gravity from micro-black hole gedanken experiment," Physics Letters B, vol. 452, no. 1-2, pp. 39-44, 1999.

[16] A. Kempf, G. Mangano, and R. B. Mann, "Hilbert space representation of the minimal length uncertainty relation," Physical Review D, vol. 52, no. 2, pp. 1108-1118, 1995.

[17] H. Hinrichsen and A. Kempf, "Maximal localization in the presence of minimal uncertainties in positions and in momenta," Journal of Mathematical Physics, vol. 37, no. 5, pp. 2121-2137, 1996.

[18] A. Kempf, "On quantum field theory with nonzero minimal uncertainties in positions and momenta," Journal of Mathematical Physics, vol. 38, no. 3, pp. 1347-1372, 1997.

[19] M. Bojowald and A. Kempf, "Generalized uncertainty principles and localization of a particle in discrete space," Physical Review D, vol. 86, no. 8, Article ID 085017, 15 pages, 2012.

[20] K. Nozari and A. Etemadi, "Minimal length, maximal momentum, and Hilbert space representation of quantum mechanics," Physical Review D, vol. 85, no. 10, Article ID 104029, 12 pages, 2012.

[21] K. Nozari and B. Fazlpour, "Some consequences of spacetime fuzziness," Chaos, Solitons \& Fractals, vol. 34, no. 2, pp. 224-234, 2007.

[22] G. Amelino-Camelia, "Relativity in spacetimes with shortdistance structure governed by an observer-independent (Planckian) length scale," International Journal of Modern Physics D, vol. 11, no. 1, pp. 35-60, 2000.

[23] G. Amelino-Camelia, "Relativity: special treatment," Nature, vol. 418, pp. 34-35, 2002. 
[24] G. Amelino-Camelia, "Doubly-special relativity: first results and key open problems," International Journal of Modern Physics D, vol. 11, no. 10, pp. 1643-1669, 2002.

[25] J. Kowalski-Glikman, "Introduction to doubly special relativity," in Planck Scale Effects in Astrophysics and Cosmology, vol. 669 of Lecture Notes in Physics, pp. 131-159, Springer, Berlin, Germany, 2005.

[26] G. Amelino-Camelia, J. Kowalski-Glikman, G. Mandanici, and A. Procaccini, "Phenomenology of doubly special relativity," International Journal of Modern Physics A, vol. 20, no. 26, pp. 6007-6037, 2005.

[27] K. Imilkowska and J. Kowalski-Glikman, "Doubly special relativity as a limit of gravity," in Special Relativity, vol. 702 of Lecture Notes in Physics, pp. 279-298, Springer, Berlin, Germany, 2006.

[28] J. Magueijo and L. Smolin, "Lorentz invariance with an invariant energy scale," Physical Review Letters, vol. 88, no. 19, Article ID 190403, 4 pages, 2002.

[29] J. Magueijo and L. Smolin, "Generalized Lorentz invariance with an invariant energy scale," Physical Review D, vol. 67, no. 4, Article ID 044017, 12 pages, 2003.

[30] J. Magueijo and L. Smolin, "String theories with deformed energy-momentum relations, and a possible nontachyonic bosonic string," Physical Review D, vol. 71, no. 2, Article ID 026010, 6 pages, 2005.

[31] J. L. Cortés and J. Gamboa, "Quantum uncertainty in doubly special relativity," Physical Review D, vol. 71, no. 6, Article ID 065015, 4 pages, 2005.

[32] A. F. Ali, S. Das, and E. C. Vagenas, "Discreteness of space fromthe generalized uncertainty principle," Physics Letters B, vol. 678, no. 5, pp. 497-499, 2009.

[33] S. Das, E. C. Vagenas, and A. F. Ali, "Discreteness of space from GUP II: relativistic wave equations," Physics Letters B, vol. 690, no. 4, pp. 407-412, 2010.

[34] P. Pedram, K. Nozari, and S. H. Taheri, "The effects of minimal length and maximal momentum on the transition rate of ultra cold neutrons in gravitational field," Journal of High Energy Physics, vol. 2011, no. 3, article 93, 2011.

[35] B. Mirza and M. Zarei, "Minimal uncertainty in momentum: the effects of IR gravity on quantum mechanics," Physical Review D, vol. 79, no. 12, Article ID 125007, 8 pages, 2009.

[36] R. Koekoek and R. F. Swarttouw, "The Askey-scheme of hypergeometric orthogonal polynomials and its q-analogue," Tech. Rep. 94-05, Faculty of Technical Mathematics and Informatics, Delft University of Technology, Delft, Netherlands, 1994.

[37] K. Nozari and Z. Soleymani, "Natural cutoffs and Hilbert space representation of quantum mechanics," in Proceedings of the Multiverse and Fundamental Cosmology (Multicosmofun '12), vol. 1514 of AIP Conference Proceedings, pp. 93-96, Szczecin, Poland, September 2012. 

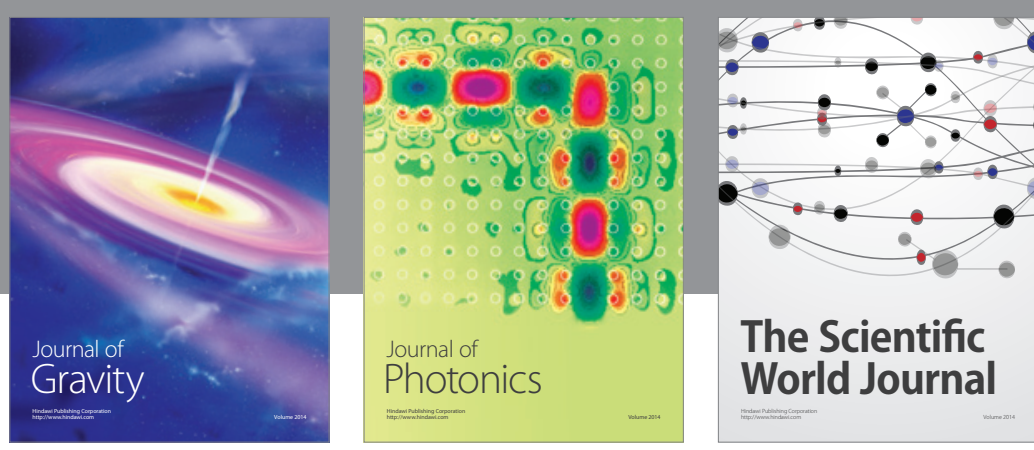

The Scientific World Journal
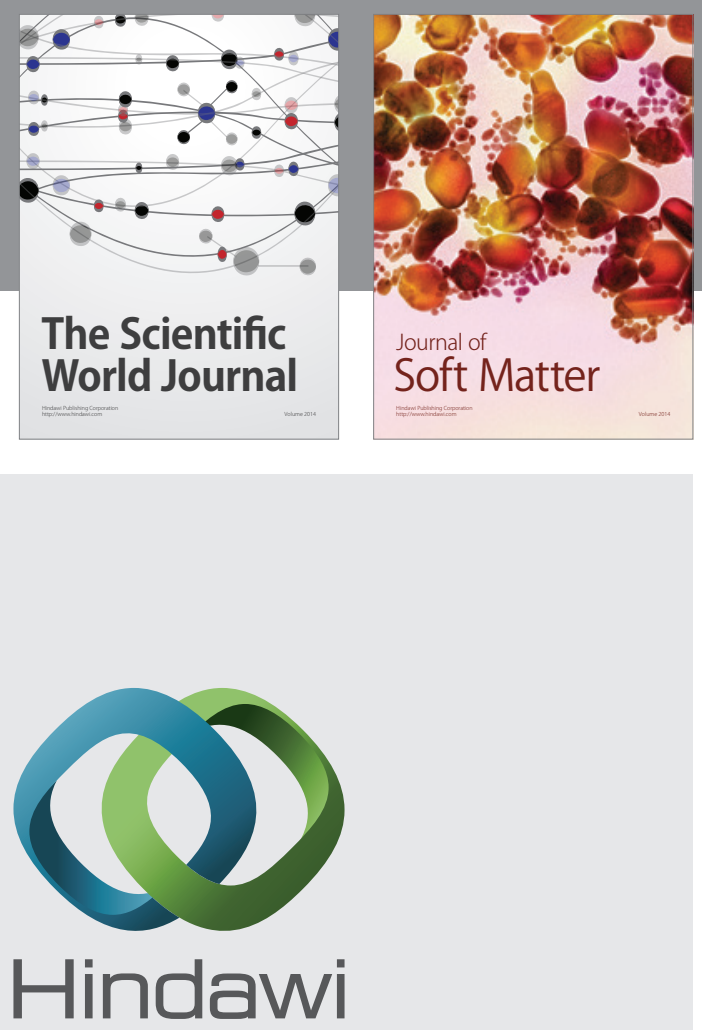

Submit your manuscripts at

http://www.hindawi.com

nternational Journal of

Statistical Mechanics
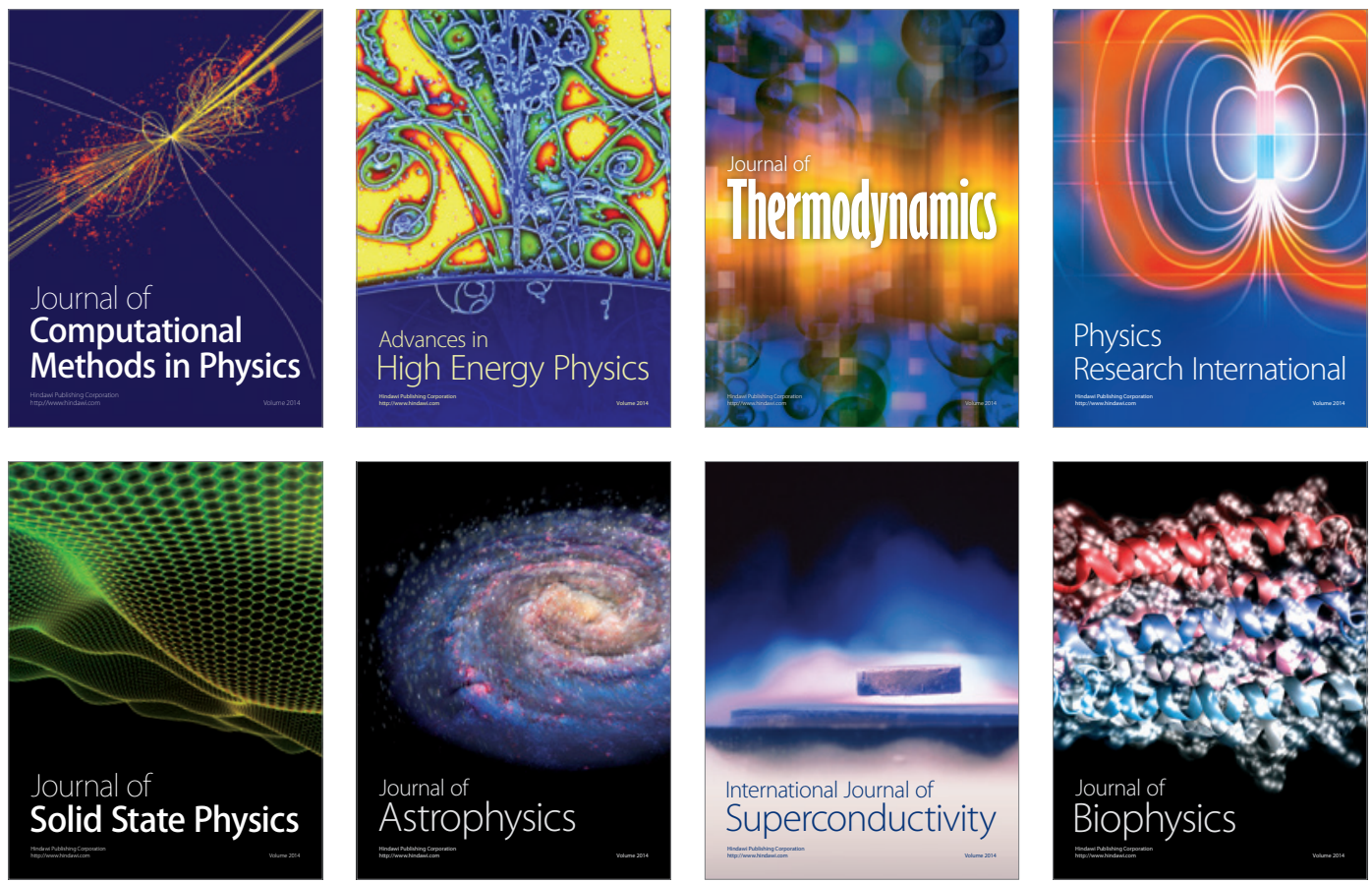
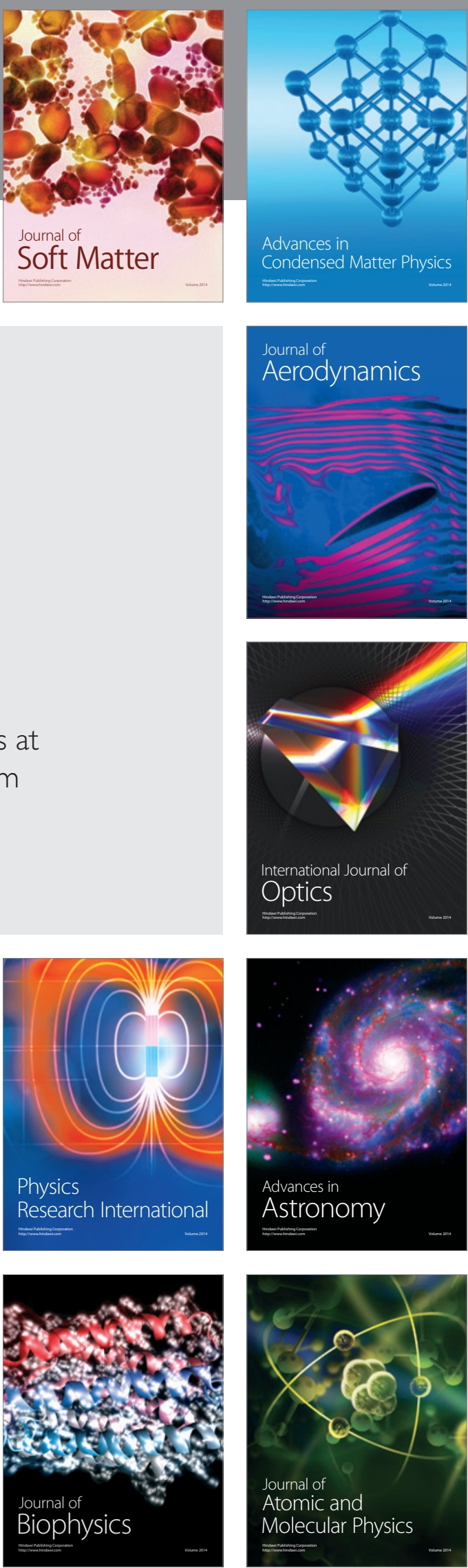